\title{
Open boundary conditions in numerical simulations of unsteady incompressible flow
}

\author{
$\begin{array}{ll}\text { M. P. Kirkpatrick } & \text { S. W. Armfield } \\ & \end{array}$
}

(Received 15 August 2008; revised 21 January 2009)

\begin{abstract}
In numerical simulations of unsteady incompressible flow, mass conservation can be enforced using a fractional step method in which the momentum equations are solved in combination with a pressure or pressure correction equation. While there is an obvious way to implement most spatial boundary conditions commonly applied to these equations, the situation for so-called open boundaries is less clear. Open boundaries must allow fluid to leave the domain with minimal effect on the upstream flow and, at the same time, ensure global conservation of mass. Previous work found that the open boundary condition implementations commonly used with lower order fractional step methods, such as the projection-1 and projection- 2 methods, can cause the higher order projection-3 method to become unstable. We discuss the implementation of open boundary conditions in combination with fractional step methods, and investigate three possible approaches.
\end{abstract}

http://anziamj . austms.org . au/ojs/index.php/ANZIAMJ/article/view/1457 gives this article, (C) Austral. Mathematical Soc. 2009. Published February 6, 2009. ISSN 1446-8735. (Print two pages per sheet of paper.) 


\section{Contents}

1 Introduction

C761

2 Time integration

C763

3 Open boundary conditions

3.1 Open boundary condition $1 \ldots \ldots \ldots \ldots$ C $\ldots \ldots 76$

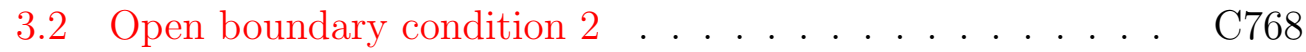

3.3 Open boundary condition $3 \ldots \ldots \ldots \ldots$ C769 . . . . . . . .

4 Test case

C770

5 Conclusions

C772

References

C772

\section{Introduction}

Fractional step projection methods integrate the Navier-Stokes equations over each time step by first solving the momentum equations using an approximate pressure field to yield an intermediate velocity field that does not, in general, satisfy conservation of mass. A Poisson equation is then solved with the divergence of the intermediate velocity as a source term to provide a pressure or pressure correction, which is then used to correct the intermediate velocity field, providing a divergence free velocity field. The pressure is updated and integration then proceeds to the next time step. An extensive list of references for various fractional step methods developed for the Navier-Stokes equations was given by Armfield and Street [2].

The projection-1 (P1) method sets the pressure field to zero in the momentum equations and the Poisson equation is then solved for the new pressure, while the projection-2 ( $\mathrm{P} 2)$ method sets the pressure in the momentum equa- 
tions to that obtained at the previous time step, and the Poisson equation is then solved for a pressure correction. Both the P1 method, with appropriate intermediate velocity boundary conditions, and the $\mathrm{P} 2$ method provide second order accuracy in time for the velocity and pressure fields, provided the momentum equations are integrated using a second order accurate scheme [1].

The projection-3 (P3) method, first proposed by Gresho [3], uses linear extrapolation from previous time steps to give a more accurate estimate of the pressure used in the momentum equations. This increases the order of the fractional step error, that is the additional error of the fractional step method with respect to an iterative scheme, from second to third order [2]. While Shen [8] showed analytically that the P3 method can lead to solutions that are unbounded in time, Armfield and Street [2] tested the method for a two dimensional natural convection flow in a square cavity and found that it remained stable for this case and reduced the size of the overall time stepping error by a factor of four. Kirkpatrick and Armfield [5] tested the P3 method for a number of more complex three dimensional flows involving different types of boundary condition. These included a large eddy simulation of a turbulent convective atmospheric boundary layer. They found that the method remained stable for all the flows tested, with the exception of cases in which an open boundary condition was used at one or more boundaries.

Kirkpatrick and Armfield [5] also showed that, by using a more accurate estimate of the pressure in the momentum equations, the P3 method significantly reduces the required number of sweeps of the matrix equation solver used to solve the pressure correction equation. Typically this reduction was $30-40 \%$ giving a reduction in run time of approximately 30\%. This is a significant advantage, warranting further research into the method.

In this article we address the issue of stability of the P3 method when used in combination with open boundary conditions. We discuss three possible approaches for implementing open boundary conditions with a fractional step time advancement scheme. We find that only one of the implementations is consistent in its representation of boundary conditions for both the momen- 
tum and pressure correction equations. Furthermore, we find that when the consistent open boundary implementation is used the P3 method remains stable.

We present results for one of our test cases - a large eddy simulation of atmospheric boundary layer flow over Mount Wellington in Tasmania. The numerical simulations are performed using the PUFFIN code $[4,6,7]$.

\section{Time integration}

The Navier-Stokes equations for incompressible flow are written in Cartesian tensor notation as

$$
\begin{aligned}
\frac{\partial u_{i}}{\partial t}+\frac{\partial u_{i} u_{j}}{\partial x_{j}} & =-\frac{\partial p}{\partial x_{i}}+\frac{1}{\operatorname{Re}} \frac{\partial^{2} u_{i}}{\partial x_{j}^{2}} \\
\frac{\partial u_{j}}{\partial x_{j}} & =0 .
\end{aligned}
$$

Here $x_{i}$ are the components of the position vector, $t$ the time, $u_{i}$ the components of the velocity vector, $p$ the pressure/density, and Re the Reynolds number. For clarity, a very simple form of the equations is used in this and the next section. All of the conclusions drawn are also applicable to the more complex equations governing the atmospheric boundary layer flow used as the test case in Section 4.

Numerical solution of the equations uses a finite volume method on a staggered Cartesian grid. All spatial derivatives are approximated using a second order central scheme. Time integration uses a hybrid implicit/explicit scheme in which Crank-Nicolson is used for the diffusion terms and a second order Adams-Bashforth scheme is used for the advection terms. The scheme is

$$
\frac{\mathbf{u}^{n+1}-\mathbf{u}^{n}}{\Delta t}+\left[\frac{3}{2} H\left(\mathbf{u}^{n}\right)-\frac{1}{2} H\left(\mathbf{u}^{n-1}\right)\right]=-G p^{n+1 / 2}+\frac{1}{2 \operatorname{Re}} L\left(\mathbf{u}^{n+1}+\mathbf{u}^{n}\right),
$$




$$
\mathrm{D} \mathbf{u}^{\mathrm{n}+1}=0
$$

where $\mathrm{H}$ is the discrete advection operator, $\mathrm{G}$ gradient, $\mathrm{L}$ diffusion and $\mathrm{D}$ divergence. Superscripts refer to time steps with the current timestep being from $n$ to $n+1$. The use of an explicit scheme for the non-linear terms ensures that the overall scheme is second order accurate in time without iteration [7].

One of the main difficulties in implementing this scheme results from the coupling between the momentum and continuity equations. Fractional step methods deal with this by dividing the integration into a series of substeps. The momentum equations are first integrated using an estimate of the pressure $\tilde{p}^{n+1 / 2}$ at time $t=n+1 / 2$, to obtain an intermediate velocity field $\tilde{u}_{i}^{n+1}$ at time $\mathrm{t}=\mathrm{n}+1$, that is

$$
\frac{\tilde{\mathbf{u}}^{\mathrm{n}+1}-\mathbf{u}^{\mathrm{n}}}{\Delta \mathrm{t}}+\left[\frac{3}{2} \mathrm{H}\left(\mathbf{u}^{\mathrm{n}}\right)-\frac{1}{2} \mathrm{H}\left(\mathbf{u}^{\mathrm{n}-1}\right)\right]=-\mathrm{G} \tilde{p}^{\mathrm{n}+1 / 2}+\frac{1}{2 \operatorname{Re}} \mathrm{L}\left(\tilde{\mathbf{u}}^{\mathrm{n}+1}+\mathbf{u}^{\mathrm{n}}\right) .
$$

In the P3 method, $\tilde{p}^{n+1 / 2}$ is obtained by extrapolating from the previous two time steps using

$$
\tilde{p}^{n+1 / 2}=2 p^{n-1 / 2}-p^{n-3 / 2} .
$$

This extrapolation is second order accurate.

In general, the intermediate velocity field obtained by integrating Equation (5) is not divergence free. In order to obtain a divergence free velocity field, a Poisson equation is solved for a pressure correction $p^{\prime}$ in which the source term is the divergence of the intermediate velocity field:

$$
\frac{\partial^{2} p^{\prime}}{\partial x_{j}^{2}}=\frac{1}{\Delta t} \frac{\partial \tilde{u}_{j}^{n+1}}{\partial x_{j}}
$$

The solution of this equation is then used to correct the pressure and velocity:

$$
\begin{aligned}
p^{n+1 / 2} & =\tilde{p}^{n+1 / 2}+p^{\prime} \\
u_{i}^{n+1} & =\tilde{u}_{i}^{n+1}-\Delta t \frac{\partial p^{\prime}}{\partial x_{i}} .
\end{aligned}
$$


The velocity correction step constitutes a projection of the intermediate velocity field onto a subspace of divergence free velocity fields. Because the approximation of the pressure $\tilde{p}^{n+1 / 2}$ used in the momentum equations is second order accurate, this projection error is third order accurate in time [2]. However, the accuracy of the overall scheme is still second order due to the second order accurate integration schemes used for the other terms in the momentum equations.

\section{Open boundary conditions}

Figure 1 shows a simple two dimensional domain with a number of different boundary conditions applied. Because the gradient of the pressure correction field is used to correct the velocity field, Equation (9), the appropriate boundary condition on the pressure correction equation depends on the boundary condition applied to the component of velocity normal to the boundary. In cases such as an inlet, a solid wall, or a zero flux boundary, a Dirichlet boundary condition is applied to the normal velocity component. In this case the velocity correction is zero and so the appropriate boundary condition for the $p^{\prime}$-equation is a Neumann boundary condition with

$$
\left.\frac{\partial p^{\prime}}{\partial n}\right|_{b}=0
$$

Open boundaries are usually applied where flow across the boundary is considered to be strongly dependent on the flow within the domain. A variety of different boundary conditions may be used; however, the most common is a simple Neumann boundary condition with the gradient set to zero:

$$
\left.\frac{\partial u_{n}}{\partial x}\right|_{b}=0
$$

We use a staggered grid with nodes arranged as shown in Figure 2. On this grid, the zero gradient velocity boundary condition above is implemented in 


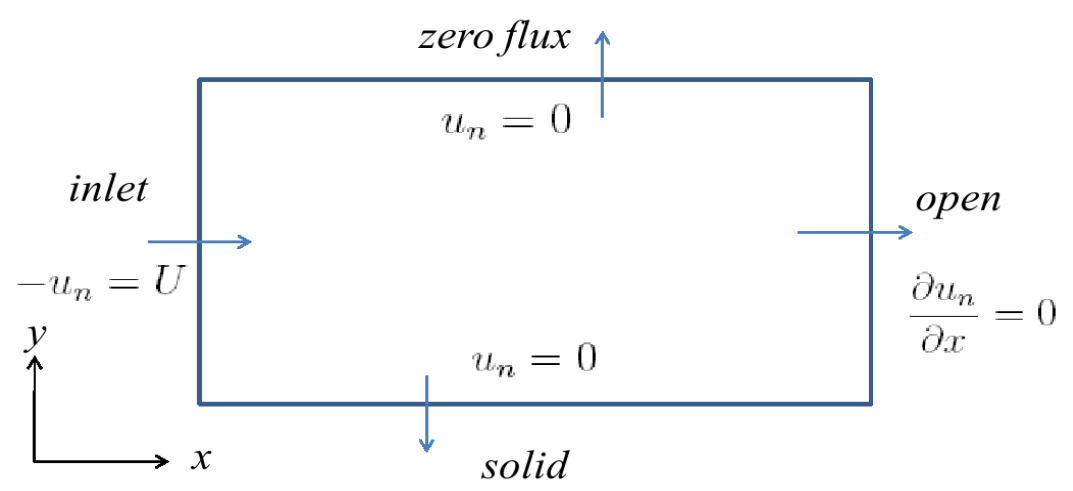

Figure 1: Simple two dimensional domain.

the discretized momentum equations, Equation (5), as

$$
\begin{aligned}
& \tilde{u}_{\mathrm{b}}=\tilde{\mathrm{u}}_{\mathrm{I}-1}, \\
& \mathrm{u}_{\mathrm{b}}=\mathrm{u}_{\mathrm{I}-1} .
\end{aligned}
$$

Given this boundary condition for $u_{n}$ we wish to determine what boundary condition to apply to the $p^{\prime}$-equation at the open boundary. The pressure correction boundary condition must satisfy a number of criteria. Firstly, the boundary condition must lead to a pressure correction field at the boundary that corrects $u_{n}$ at the boundary such that global conservation of mass is enforced. Secondly, since the pressure correction equation is a Poisson equation, the boundary conditions applied must satisfy the Cauchy condition, which states that an equation of the form

$$
\frac{\partial^{2} \pi}{\partial x^{2}}+\frac{\partial^{2} \pi}{\partial y^{2}}=f(x, y)
$$

has a solution on the domain $0 \leq x \leq X$ and $0 \leq y \leq Y$ only if the integrals of both sides of the equation over the domain are equal, that is if

$$
\int_{0}^{X} \int_{0}^{Y} \frac{\partial^{2} \pi}{\partial x^{2}}+\frac{\partial^{2} \pi}{\partial y^{2}} d x d y=\int_{0}^{X} \int_{0}^{Y} f(x, y) d x d y .
$$




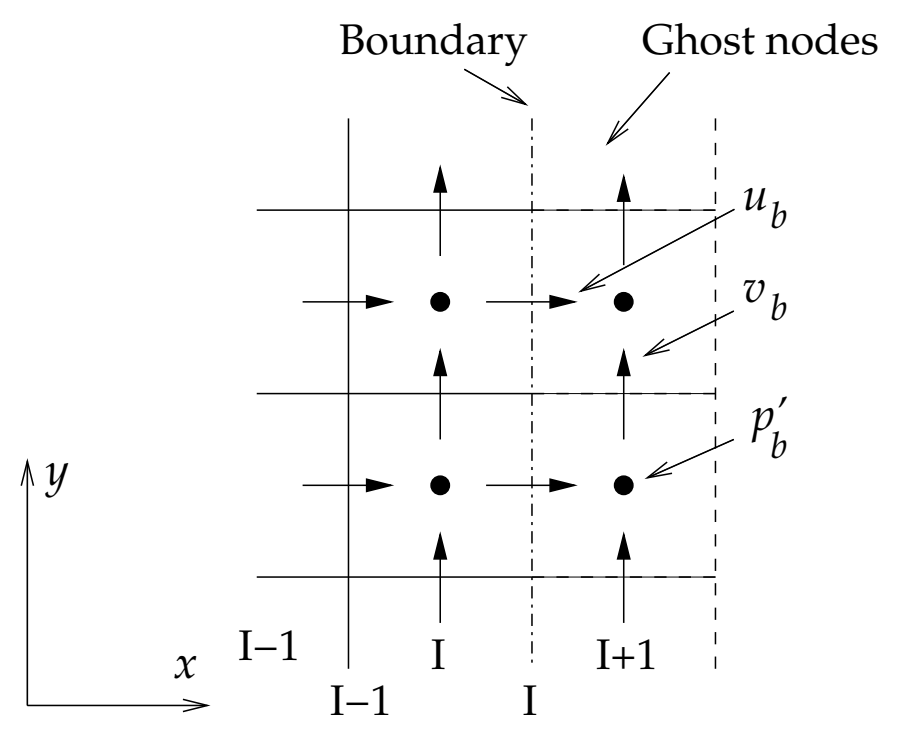

Figure 2: Position of grid nodes close to a boundary.

Using the divergence theorem, this condition is rewritten as

$$
\int_{0}^{Y}\left(\left.\frac{\partial \pi}{\partial x}\right|_{x=X}-\left.\frac{\partial \pi}{\partial x}\right|_{x=0}\right) d y+\int_{0}^{X}\left(\left.\frac{\partial \pi}{\partial y}\right|_{y=Y}-\left.\frac{\partial \pi}{\partial y}\right|_{y=0}\right) d x=\int_{\Omega} f(x, y) d \omega .
$$

The third criterion is that the $p^{\prime}$-equation boundary condition should be consistent with the boundary condition applied to the momentum equations, in the sense that it leads to a corrected velocity field that satisfies Equation (13). In the following subsections we assess three possible choices of boundary condition for the $\mathrm{p}^{\prime}$-equation at open boundaries in light of these criteria.

\subsection{Open boundary condition 1}

One possible choice is a Dirichlet boundary condition

$$
p_{\mathrm{b}}^{\prime}=0 \text {. }
$$


Consider the problem shown in Figure 1. A Dirichlet boundary condition is applied to the normal component of velocity at all boundaries other than the open boundary, so the boundary condition for the $p^{\prime}$-equation on these boundaries is

$$
\left.\frac{\partial p^{\prime}}{\partial n}\right|_{b}=0 .
$$

The Cauchy condition applied to the $p^{\prime}$-equation then becomes

$$
\left.\int_{0}^{\gamma} \frac{\partial p^{\prime}}{\partial x}\right|_{b} d y=\frac{1}{\Delta t} \int_{\Omega} \frac{\partial \tilde{u}_{j}}{\partial x_{j}} d \omega .
$$

With

$$
u_{i}^{\prime}=-\Delta t \frac{\partial p^{\prime}}{\partial x_{i}}
$$

this gives

$$
\int_{0}^{Y} u_{b}^{\prime} d y=-\int_{\Omega} \frac{\partial \tilde{u}_{j}}{\partial x_{j}} d \omega .
$$

From this we conclude that the boundary condition satisfies the Cauchy condition and also gives a correction to the velocity at the open boundary that ensures that global mass conservation is enforced. Thus two of the three criteria set out above are satisfied. However, the third criterion-that the $p^{\prime}$-equation boundary condition be consistent with the velocity boundary condition - is not satisfied, since the pressure correction step results in a final velocity field in which $\mathfrak{u}_{\mathfrak{b}} \neq \mathfrak{u}_{\mathrm{I}-1}$, which contradicts Equation (13).

\subsection{Open boundary condition 2}

A second approach involves correcting the open boundary velocity before the pressure correction step. The divergence of the intermediate velocity field is integrated over the domain and used to determine a bulk correction required to give global mass conservation:

$$
u_{b}^{\prime}=-\frac{1}{Y} \int_{\Omega} \frac{\partial \tilde{u}_{j}}{\partial x_{j}} d \omega .
$$


This correction is then applied uniformly to the boundary velocity nodes:

$$
\mathrm{u}_{\mathrm{b}}=\tilde{\mathrm{u}}_{\mathrm{b}}+\mathrm{u}_{\mathrm{b}}^{\prime} .
$$

Since the boundary velocities have now been corrected the appropriate boundary condition for the $p^{\prime}$-equation is

$$
\left.\frac{\partial p^{\prime}}{\partial x}\right|_{\mathrm{b}}=0 .
$$

This approach also satisfies the Cauchy condition and enforces global mass conservation. However, as with the previous one, $\mathfrak{u}_{\mathrm{b}} \neq \mathfrak{u}_{\mathrm{I}-1}$, indicating that this boundary condition is not consistent with the velocity boundary condition.

\subsection{Open boundary condition 3}

In order to derive a boundary condition for $p^{\prime}$ that is consistent with the velocity boundary condition, we note that if both the intermediate and final velocity fields are to satisfy the Neumann boundary condition, that is

$$
\left.\frac{\partial u}{\partial x}\right|_{b}=0 \quad \text { and }\left.\quad \frac{\partial \tilde{u}}{\partial x}\right|_{b}=0 \text {, }
$$

then so too must the velocity correction,

$$
\left.\frac{\partial u^{\prime}}{\partial x}\right|_{b}=0 \text {. }
$$

Combining this equation with Equation (9) gives the a boundary condition for $p^{\prime}$ :

$$
\left.\frac{\partial^{2} p^{\prime}}{\partial x^{2}}\right|_{b}=0 .
$$

This boundary condition must lead to $\mathfrak{u}_{\mathrm{b}}=\mathfrak{u}_{\mathrm{I}-1}$, since this is implicit in its derivation. It is also simple to show that it also satisfies the Cauchy 
condition and enforces conservation of mass. The only issue is that, because the gradient of the pressure correction on the east and west faces of the Ith row of cells must be the same, the associated coefficients $a_{E}$ and $a_{W}$ in the discrete equation become zero. As a result the solution in the last row of cells simply becomes independent of the solution in the remainder of the domain. We found this not to cause a problem as long as there is no other communication between the two parts of the domain.

\section{Test case}

The three boundary conditions discussed in the previous section were tested for a number of cases. While simulations using the P3 method quickly became unstable when boundary conditions 1 and 2 were used, they remained stable with boundary condition 3. Here we present results for one of the test cases - a large eddy simulation of atmospheric boundary layer flow over Mount Wellington, Tasmania. A figure showing the domain, grid and boundary conditions used for this simulation are attached as supplementary material. A movie and still images visualizing the flow are also attached.

The governing equations are the Boussinesq equations for a shallow atmospheric boundary layer. The simulations were run on a $16 \mathrm{CPU}$ parallel machine, with parallelization achieved using MPI. Details of the equations, matrix solver, computer architecture and parallelization are given by Kirkpatrick and Armfield [5].

The simulation was run to $7200 \mathrm{~s}$ without any stability problems. Figure 3 shows the time error plotted against time step size for $t=3600 \mathrm{~s}$ to $3603 \mathrm{~s}$. These results demonstrate that the overall scheme is second order accurate in time as expected. 


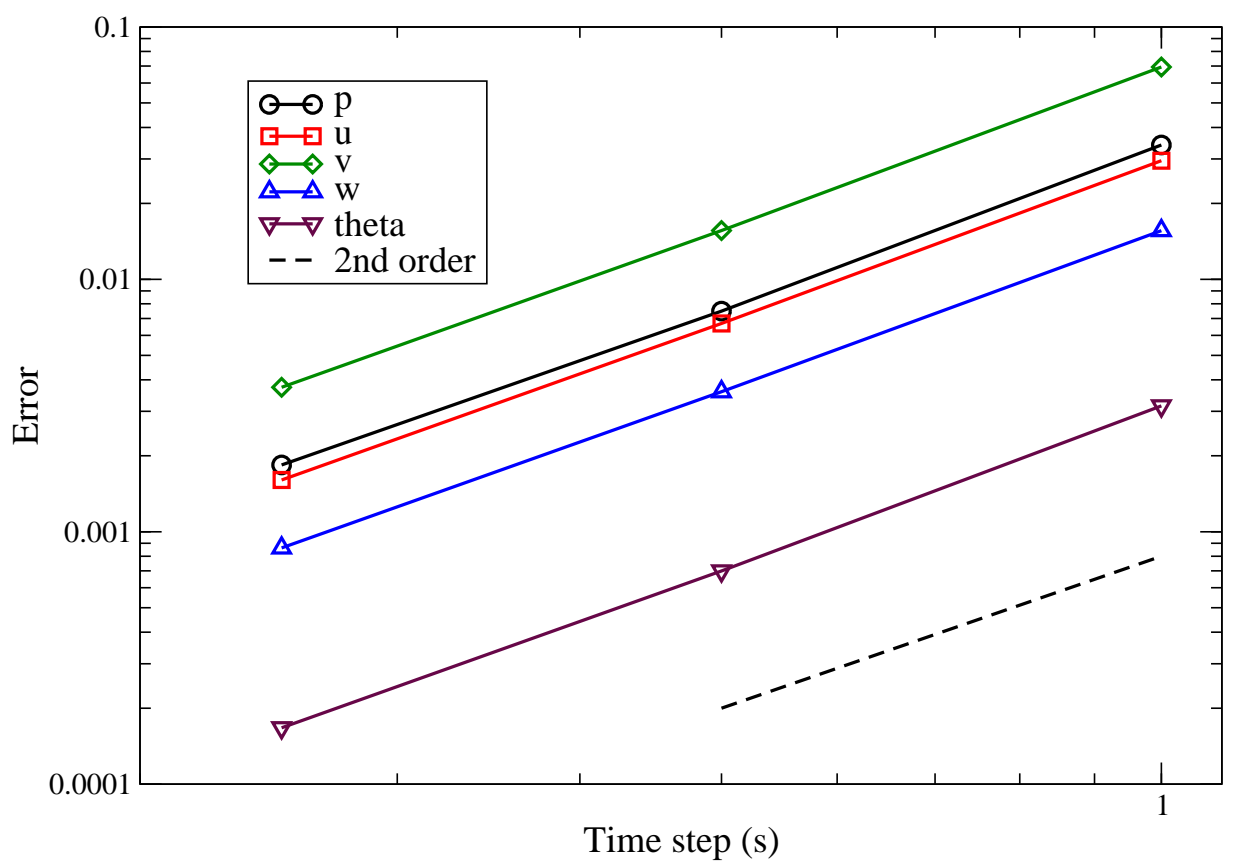

Figure 3: Variation of time error with time step size. 


\section{Conclusions}

Three open boundary conditions for the pressure correction equation were analysed and tested with the P3 fractional step method. Two of the three boundary conditions are inconsistent with the zero gradient velocity boundary condition. The third boundary condition, which imposes a requirement of zero curvature in the pressure correction field across the boundary, is consistent with the zero gradient velocity boundary condition. It also satisfies the Cauchy condition, and enforces global conservation of mass. While simulations using the first two boundary conditions remain stable with lower order fractional step methods, they become unstable when the third order P3 method is used. Simulations using the zero curvature boundary condition with the P3 method remained stable for all cases tested.

\section{References}

[1] S. W. Armfield and R. Street. An analysis and comparison of the time accuracy of fractional-step methods for the Navier-Stokes equations on staggered grids. Int. J. Numer. Meth. Fluids, 38:255-282, 2002. doi:10.1002/fld.217. C762

[2] S. W. Armfield and R. Street. Modified fractional-step methods for the Navier-Stokes equations. ANZIAM J., 45:C364-C377, 2004. http://anziamj. austms.org.au/ojs/index.php/ANZIAMJ/article/ view/894. C761, C762, C765

[3] P. Gresho. On the theory of semi-implicit projection methods for viscous incompressible flow and its implementation via finite element method that also introduces a nearly consistent mass matrix. Part 1: Theory. Int. J. Numer. Meth. Fluids, pages 587-620, 1990. doi:10.1002/fld.1650110509. C762 
[4] M. P. Kirkpatrick. A large eddy simulation code for industrial and environmental flows. PhD thesis, The University of Sydney, 2002. C763

[5] M. P. Kirkpatrick and S. W. Armfield. On the stability and performance of the projection-3 method for the time integration of the Navier-Stokes equations. ANZIAM J., 49:C559-C575, 2008. http://anziamj . austms .org.au/ojs/index.php/ANZIAMJ/article/ view/307. C762, C770

[6] M. P. Kirkpatrick, S. W. Armfield, and J. H. Kent. A representation of curved boundaries for the solution of the Navier-Stokes equations on a staggered three-dimensional Cartesian grid. J. Comp. Phys., 184:1-36, 2003. doi:10.1016/S0021-9991(02)00013-X. C763

[7] M. P. Kirkpatrick, S. W. Armfield, J. H. Kent, and T. Dixon. Simulation of vortex-shedding flows using high-order fractional step methods. ANZIAM J., 42(E):856-876, 2000. http://anziamj .austms . org.au/ojs/index.php/ANZIAMJ/article/view/625. C763, C764

[8] J. Shen. A remark on the projection-3 method. Int. J. Numer. Meth. Fluids, 16:249-253, 1993. doi:10.1002/fld.1650160308. C762

\section{Author addresses}

1. M. P. Kirkpatrick, School of Aerospace, Mechanical \& Mechatronic Engineering, University of Sydney, Sydney, Australia. mailto:michael . kirkpatrick@aeromech . usyd. edu . au

2. S. W. Armfield, School of Aerospace, Mechanical \& Mechatronic Engineering, University of Sydney, Sydney, Australia. 